\title{
Brief Study on Antibiotics Rifampin, Ethambutol, Pyrazinamide. Isoniazid for the Treatment of Tuberculosis Patient; A Combination Therapy, Advanced Techniques.
}

\author{
Umama Yezdani ${ }^{* *}$, Raja Jatav², Vishal Kumar ${ }^{1}$, Priyanka Priya², Mayur Sadar ${ }^{4}$ \\ ${ }^{1}$ Department of Pharmacy Practice, MMDU University, Ambala Haryana, india. \\ ${ }^{2}$ Department of Pharmacy,Truba Institute of Pharmacy, Bhopal Madhya Pradesh, India. \\ ${ }^{3}$ Department of Pharmacy Practice, MRM College of pharmacy hyderabad Telangana India \\ ${ }^{4}$ Department of Pharmacy Anuradha College of Pharmacy, Chikhli Maharashtra India
}

*Corresponding Author: Umama Yezdani, Department of Pharmacy Practice MRM College of Pharmacy Hyderabad.

\begin{abstract}
Antibiotics is nowadays being using for treatment of various disease and tuberculosis is one of the most diseases. TB is basically two types and their period is varying person to person. Consumption it was Potentially serious bacterial disease that effects the lungs basically. TB are spread when an infected person coughs or sneezes. Treatable by a medical professional It was Spreads easily, It Requires a medical diagnosis, Lab tests or imaging always required There is basically two types of tuberculosis latent TB, Active TB Respectively. In this article we explained results and justifiable conclusions as per outcome patient counselling, ADR Reports, others report and past and Present Research/Review Article.
\end{abstract}

Keywords: Tuberculosis, Antibiotics, Neural Structure, Drug therapy.

\section{INTRODUCTION}

Doctors prescribe antibiotics to kill the bacteria that cause it. need to take them for 6 to 9 months. What medications you take and how long people have to take them depends on which works to eradicate people TB. Sometimes, antibiotics used to treat the disease don't work. Doctors call this "drug-resistant" TB. If people have this form of the disease, people may need to take stronger medications for longer.[1]

It is commonly known as Consumption. It can spread to other parts of your body, like your brain and spine. A type of bacteria called Mycobacterium tuberculosis causes it. WHO fact sheet dated March 2010 on tuberculosis stated that overall one third of the world's population (over 2 billion) is currently infected with the TB bacillus. According to it, every second, someone in the world is newly infected with TB bacilli and 1 in every 10 of these newly infected people will become sick or infectious later in life. Since concurrent infection with HIV weakens the immune system, people with co-infection of HIV and TB are much more likely to develop TB; it is a leading cause of death among HIV-positive people. In Africa, HIV is the single most important factor contributing to the increase in the incidence of TB since 1990. The same fact sheet stated that in 2008, globally speaking, there were 9.37 million new cases of TB, with the African region and the Southeast Asian region (SEAR) having a share of 30\% and 34\%, respectively. However, the estimated incidence rate in Sub-Saharan Africa is nearly twice that in the SEAR with over 350 cases per 100,000 individuals of the human population. In the same year 2008, an estimated 1.3 million people died from TB. The highest number of deaths was in SEAR, while the highest mortality per capita was in the African region.

\subsection{Tuberculosis}

Tuberculosis (TB) is an infectious disease usually caused by Mycobacterium tuberculosis (MTB) bacteria. Tuberculosis generally affects the lungs, but can also affect other parts of the body. 
Brief Study on Antibiotics Rifampin, Ethambutol, Pyrazinamide. Isoniazid for the Treatment of Tuberculosis Patient; A Combination Therapy, Advanced Techniques

\subsubsection{Tuberculosis Types}

A TB infection doesn't mean get sick. There are two forms of the disease:

Latent TB. If the germs in body, the immune system stops them from spreading. That means doesn't have any symptoms and not contagious. But the infection is still alive into the body and can one day become active. treat with antibiotics to lower the risk for developing active TB.

The bacteria remain in the body in an inactive state. They cause no symptoms and are not contagious, but they can become active.

Active TB. This means the germs multiply and can make sick. It can spread the disease to others. Ninety percent of adult cases of active TB are from the reactivation of a latent TB infection. the bacteria do cause symptoms and can be transmitted to others. About one-third of the world's population is believed to have latent TB. There is a 10 percent chance of latent TB becoming active, but this risk is much higher in people who have compromised immune systems, i.e., people living with HIV or malnutrition, or people who smoke. TB affects all age groups and all parts of the world. However, the disease mostly affects young adults and people living in developing countries. In 2012, 80 percent of reported TB cases occurred in just 22 countries.

\subsection{Tuberculosis Diagnosis}

There are two common tests for tuberculosis, but they don't tell confirmed whether you have latent or active TB:

- Skin test. This is also known as the Mantoux tuberculin skin test. A health care worker injects a small amount of fluid into the skin and lower arm. After 2 or 3 days, they will check for swelling in arm to determine results. If results are positive, probably have been infected with TB bacteria. But the results can be false positive. If gotten a tuberculosis vaccine called bacillus Calmette-Guerin (BCG), the test could say have TB when really don't. The results can also be false negative, saying that don't have TB when really do, if infection is recent. might get this test more than once.

- Blood test. These tests, also called interferon-gamma release assays or IGRAs, measure the response when TB proteins are mixed with a small amount of blood.

The most common diagnostic test for TB is a skin test where a small injection of PPD tuberculin, an extract of the TB bacterium, is made just below the inside forearm. The injection site should be checked after 2-3 days, and, if a hard, red bump has swollen up to a specific size, then it is likely that TB is present. Unfortunately, the skin test is not 100 percent accurate and has been known to give incorrect positive and negative readings.

However, there are other tests that are available to diagnose TB. Blood tests, chest X-rays, and sputum tests can all be used to test for the presence of TB bacteria and may be used alongside a skin test. Multidrug-resistant TB (MDR-TB) is more difficult to diagnose than regular TB. It is also difficult to diagnose regular TB in children.

\subsection{Cause}

The cause of TB is infection of human tissue(s) by the bacterium Mycobacterium tuberculosis (mycobacteria or TB). These bacteria are slow growing, aerobic, and can grow within body cells (an intracellular parasitic bacterium). Its unique cell wall helps protect it from the body's defenses and gives mycobacteria the ability to retain certain dyes like fuchsin (a reddish dye) after an acid rinse that rarely happens with other bacterial, fungal, or parasitic genera.

Mycobacteria that escape destruction by body defenses may be spread by blood or lymphatic pathways to most organs, with preference to those that oxygenate well (lungs, kidneys, and bones, for example). Typical TB lesions, termed granulomas, usually consist of a central necrotic area, then a zone with macrophages, giant Langerhans cells and lymphocytes that become surrounded by immature macrophages, plasma cells, and more lymphocytes. These granulomas also contain mycobacteria. In latent infections, a fibrous capsule usually surrounds the granulomas, and in some people, the granulomas calcify, but if the immune defenses fail initially or at a later time (reactivate), the bacteria continue to spread and disrupt organ functions. 


\subsubsection{What are the Risk Factors for Tuberculosis?}

There are many risk factors for developing tuberculosis. Certain groups of people have a high risk, such as people who work in hospitals and other areas where TB-infected people may reside (jails, nursing homes, group homes for HIV patients, homeless shelters). Close association with drug users, or people with known TB infections are also at higher risk. Other people at high risk include the following:

Visitors and immigrants from areas known to have high incidence of TB

Children and the elderly with weakened immune systems (especially those with a positive TB skin test, see below)

- Patients with HIV infection

- Drug abusers, especially IV drug abuse

- Head and neck cancer patients

- Transplant patients

- Diabetics

- Kidney disease patients

- People undergoing immunosuppressive therapy

- Silicosis

1.3.2. Symptoms of Tuberculosis

Although there are a number of TB types, pulmonary tuberculosis is responsible for the majority (about $85 \%$ ) of TB infections. Consequently, pulmonary TB symptoms and signs may occur with or even before other types of TB are diagnosed. The classical clinical symptoms and signs of pulmonary TB may include the following:

- Fever

- Night sweats

- Cough (often chronic)

- Hemoptysis (coughing up bloody sputum)

- Decrease or loss of appetite

- Weight loss and/or muscle loss (unintentional)

- Fatigue and/or malaise

- Chest pain (pain while breathing)

- Shortness of breath

- Swollen lymph nodes

- Pneumonitis (may be the only symptom in the elderly).

Other types are loosely classified as extra pulmonary and often have symptoms that are nonspecific but frequently localized to the involved site. The following includes the signs and symptoms of additional types of TB:

- Skeletal TB (also termed Pott's disease): spinal pain, back stiffness, paralysis is possible

- $\quad$ TB meningitis: headaches (variable in length but persistent), mental changes, coma

- TB arthritis: usually pain in a single joint (hips and knees most common)

- Genitourinary TB: dysuria, flank pain, increased frequency, masses or lumps (granulomas)

- Gastrointestinal TB: difficulty swallowing, nonhealing ulcers, abdominal pain, malabsorption, diarrhea (may be bloody) 
Brief Study on Antibiotics Rifampin, Ethambutol, Pyrazinamide. Isoniazid for the Treatment of Tuberculosis Patient; A Combination Therapy, Advanced Techniques

- Miliary TB: many small nodules widespread in organs that resemble millet seeds (hence its name)

- Pleural TB: empyema and pleural effusions

- Multidrug-resistant tuberculosis (MDR TB): patients infected with TB bacteria that are resistant to multiple drugs

- XDR TB: patients infected with TB bacteria that are resistant to some of the most effective anti-TB medications; XDR stands for extensively drug resistant

- Caseous TB: Necrotic (dead and dying) tissue that is infected with TB organisms has a soft, dry, and cheesy appearance.

- End-stage TB: Patients may suffer fever, constant coughing, shortness of breath, weight loss, mental changes, and blood in their sputum before death.

\subsection{Treatment}

The majority of TB cases can be cured when the right medication is available and administered correctly. The precise type and length of antibiotic treatment depend on a person's age, overall health, potential resistance to drugs, whether the TB is latent or active, and the location of infection (i.e., the lungs, brain, kidneys). People with latent TB may need just one kind of TB antibiotics, whereas people with active TB (particularly MDR-TB) will often require a prescription of multiple drugs.Antibiotics are usually required to be taken for a relatively long time. The standard length of time for a course of TB antibiotics is about 6 months.TB medication can be toxic to the liver, and although side effects are uncommon, when they do occur, they can be quite serious. Potential side effects should be reported to a doctor and include:

- Dark urine - colour of the urine and potentially lead to darker urine are carotene, food colorings, beets, blackberries, laxatives, B complex vitamins, and drugs such as Pyridium. The urine may also appear darker when there is bleeding into the urine, and the numerous causes of bloody urine (for example, infections, stones, cancer, trauma, kidney diseases) can all contribute to a darker appearance of the urine. In the presence of a urinary tract infection

- Fever - Fever is when a human's body temperature goes above the normal range of $36-37^{\circ}$ Centigrade $\left(98-100^{\circ}\right.$ Fahrenheit). It is a common medical sign. Other terms for a fever include pyrexia and controlled hyperthermia.

- Loss of appetite - A decreased appetite occurs when you have a reduced desire to eat. It may also be known as a poor appetite or loss of appetite. The medical term for this is anorexia

- Nausea and vomiting - The timing of the nausea or vomiting can indicate the cause. When appearing shortly after a meal, nausea or vomiting may be caused by food poisoning, gastritis (inflammation of the stomach lining), an ulcer, or bulimia. Nausea or vomiting.

- It is important for any course of treatment to be completed fully, even if the TB symptoms have gone away. Any bacteria that have survived the treatment could become resistant to the medication that has been prescribed and could lead to developing MDR-TB in the future. Extensively drugresistant TB (XDR TB) is resistant to some first- and second-line antibiotics. It is resistant to the most powerful TB drugs and is hard to treat. This makes it a serious risk for people with HIV and other conditions that weaken the immune system.

- Directly observed therapy (DOT) may be recommended. This involves a healthcare worker administering the TB medication to ensure that the course of treatment is completed.

Table1. Latent TB Infection Treatment Regimens

\begin{tabular}{|c|c|c|c|}
\hline Drugs & Duration & Interval & Minimum Doses \\
\hline Isoniazid & 9 months & Daily Twice weekly & 27076 \\
\hline Rifampin & 4 months & Daily & 120 \\
\hline
\end{tabular}

\subsubsection{Antibiotics}

Antibiotics play great role for the treatment of tuberculosis these are few categorized drugs used for treatment propose of tuberculosis are rifampicin, isoniazid 


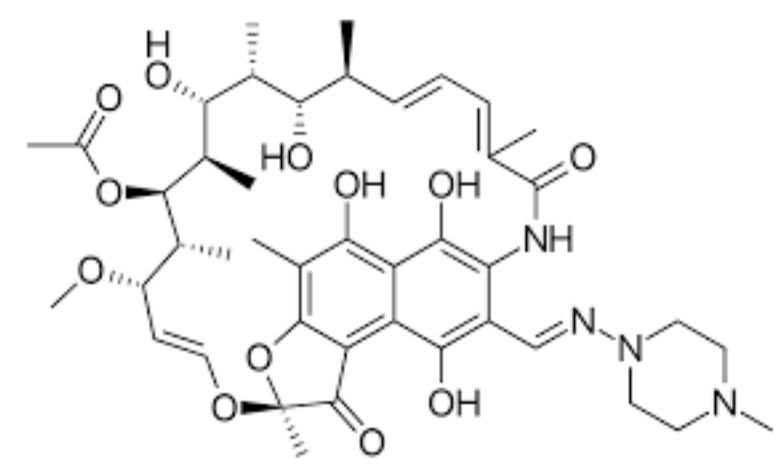

Figure1.

Rifampicin, also known as rifampin, is an antibiotic used to treat several types of bacterial infections, including tuberculosis, Mycobacterium avium complex, leprosy, and Legionnaires' disease.

\subsubsection{Pyrazinamide}

Pyrazinamide kills or stops the growth of certain bacteria that cause tuberculosis (TB). It is used with other drugs to treat tuberculosis. It is a highly specific agent and is active only against Mycobacterium tuberculosis. In vitro and in vivo, the drug is active only at a slightly acid $\mathrm{pH}$. Pyrazinamie gets activated to Pyrazinoic acid in the bacilli where it interferes with fatty acid synthase FAS I. This interferes with the bacteriums ability to synthesize new fatty acids, required for growth and replication.

\subsubsection{Mechanism of Action}

Pyrazinamide diffuses into active $M$. tuberculosis that express pyrazinamidase enzyme that converts pyrazinamide to the active form pyrazinoic acid. Pyrazinoic acid can leak out under acidic conditions to be converted to the protonated conjugate acid, which is readily diffused back into the bacilli and accumulate intracellularly. The net effect is that more pyrazinoic acid accumulates inside the bacillus at acid $\mathrm{pH}$ than at neutral $\mathrm{pH}$. Pyrazinoic acid was thought to inhibit the enzyme fatty acid synthase (FAS) I, which is required by the bacterium to synthesise fatty acids. However, this theory was thought to have been discounted. However, further studies reproduced the results of FAS I inhibition as the putative mechanism first in whole cell assay of replicating M. tuberculosis bacilli which have shown that pyrazinoic acid and its ester inhibit the synthesis of fatty acids. This study was followed by in vitro assay of tuberculous FAS I enzyme that tested the activity with pyrazinamide, pyrazinoic acid and several classes of pyrazinamide analogs. Pyrazinamide and its analogs inhibited the activity of purified FAS . It has also been suggested that the accumulation of pyrazinoic acid disrupts membrane potential and interferes with energy production, necessary for survival of $\mathrm{M}$. tuberculosis at an acidic site of infection. Pyrazinoic acid has also been shown to bind to the ribosomal protein S1 (RpsA) and inhibit trans-translation. This may explain the ability of the drug to kill dormant mycobacteria.

- Absorption - Rapidly and well absorbed from the gastrointestinal tract.

- Protein binding - 10\% (bound to plasma proteins)

- Metabolism - Hepatic, Pyrazinamide 5-hydroxypyrazinamide

- Route of elimination - Approximately $70 \%$ of an oral dose is excreted in the urine, mainly by glomerular filtration within 24 hours

- Half-life - 9-10 hours (normal conditions)

- Toxicity - Side effects include liver injury, arthralgias, anorexia, nausea and vomiting, dysuria,malaise and fever, sideroblastic anemia, adverse effects on the blood clotting mechanism or vascular integrity, and hypersensitivity reactions such as urticaria, pruritis and skin rashes.

- Affected organisms - Mycobacterium tuberculosis 


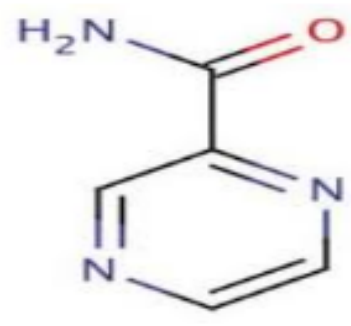

Figure2.

- Isoniazid is used with other medications to treat active tuberculosis (TB) infections. It is also used alone to prevent active TB infections in people who may be infected with the bacteria (people with positive TB skin test). Isoniazid is an antibiotic and works by stopping the growth of bacteria.

- Ethambutol - Ethambutol HCL. ... Ethambutol is used with other medications to treat tuberculosis (TB). ... Continue to take this medication (and other TB medications) until the full prescribed amount is finished, even if symptoms disappear.

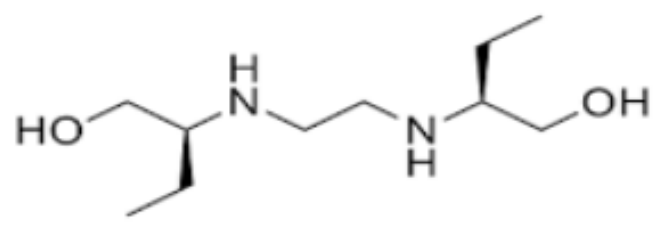

Figure3.

\section{Materials And Methods}

In order to extract current trends in diagnosis and medical or surgical treatment of spinal TB we performed a narrative review with analysis of all the articles available to us published between the years 2009 and 2019.

The reports about different aspects of spinal TB, with acceptable design, clearly explained results and justified conclusions according to the data were included in this review. Since, one of the aims of this article was to discuss the historical aspects of TB management we included articles regardless of their time of publication.

\subsection{Collection of Data}

Data is collected from, Gandhi Medical College Indore MP

\subsection{Pathophysiology \& Radio diagnosis}

- There is evidence of area of collapse/consolidation with few areas of adjacent fibrosis is seen in the apical segment of right lower lobe with associated varicose bronchiectasis is seen in this region. Mild thickening of oblique fissure is seen in posterior aspect. No evidence of any mass lesion seen. No evidence of bronchial occlusion seen.

- Rest of the bilateral lung fields appearly grossly clear.

- No e/o cavitation is seen on either side.

- NO e/o any centrilobular nodules seen.

- No e/o ground glass haze/septal thickening seen.

- No e/o honey combing is seen.

- Small hypodense lymph nodes are seen in right hilar region measuring approx. $10 \mathrm{~mm}$.

- No e/o pleural effusion is seen on either side.

- Treachea and rest of the major bronchi are normal. 
- Mediastinal vessels and heart shows normal contrast opacification.

- Visualised part of abdomen appears normal.

\subsection{Impression}

An area of collapse or consolidation in apical segment of right lower lobe with adjacent fibrosis with associated varicose type of bronchiectasis likely infective etiology. Advice clinical correlation.

Ms. Geeta Tiwari have spondylodiscitis TB in figure 4 finish which is shown clearly the Patient Condition in X Ray Report.

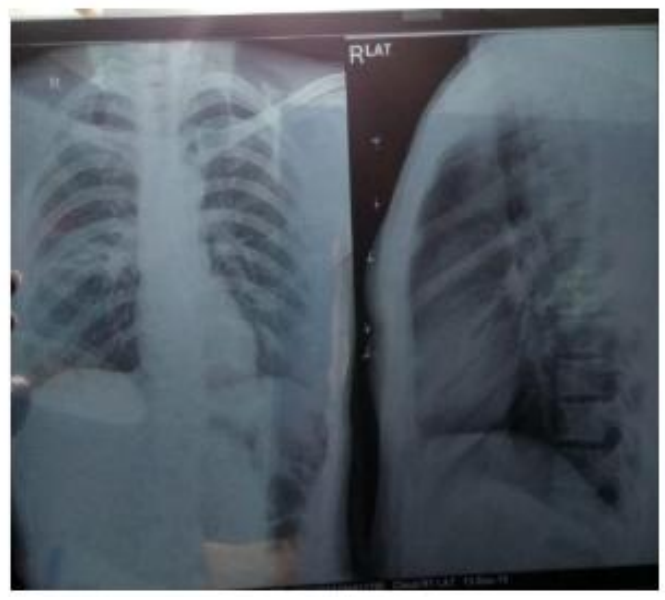

Figure4. X Ray Report of Geeta Tiwari

\subsection{Biochemistry}

Table2.

\begin{tabular}{|c|c|c|}
\hline TEST NAME & RESULT & NORMAL RANGE \\
\hline Total Bilirubin & $3.06 \mathrm{mg} / \mathrm{dl}$ & $0-1.00 \mathrm{mg} / \mathrm{dl}$ \\
\hline Direct Bilirubin & $0.97 \mathrm{mg} / \mathrm{dl}$ & $0.2-04 \mathrm{mg} / \mathrm{dl}$ \\
\hline Indirect Bilirubin & $1.8 \mathrm{mg} / \mathrm{dl}$ & $0.0-0.75 \mathrm{mg} / \mathrm{dl}$ \\
\hline
\end{tabular}

\subsection{Therapy}

Combined Drug Therapy (DOT), Thrice a day rifampicin, isoniazid and other antibiotics for the treatment of active tuberculosis.

\subsection{Mri- Brain with Contrast}

\subsubsection{Techniques}

MRI of brain was performed using T1, T2, Flair, axial SWI and diffusion weight sequences in focus planes. Post Contracting Study using T1W sequences in various planes.

\section{RESUlTS \& DisCUSSION}

In a Patient of pulmonary tuberculosis presented with seizures, above MRI Findings of Multiple ring enhancing lessions with perifocal edema suggest possibility of tuberculosis.

\section{CONCLUSiON}

Tuberculosis is very serious disorder overall Discussion and as Per X Ray, MRI, CT Scan Reports, and overall findings of patient TB suggested possibility of tuberculosis. Ms Geeta is suffering a series problem such as pain in spinal cord and Neuro disturbance. The DOT therapy is suggested for more 68 month's including Antibiotics; rifampicin because rifampicin antibiotic used to treat several types of bacterial infections, including tuberculosis, Mycobacterium avium .

\section{ACKNOWLEDGEMENT}

First of all, I'm thankful to Md Gayoor khan sir [ My Mentor] for proper guidance and encouragement time to time. 
Brief Study on Antibiotics Rifampin, Ethambutol, Pyrazinamide. Isoniazid for the Treatment of Tuberculosis Patient; A Combination Therapy, Advanced Techniques

ABbreViation

TB - Tuberculosis, NMD - Neuro motor disease, BI - Bacterial infection

\section{REFERENCES}

[1] Mohammad Gayoor Khan ,Umama Yezdani, Shivam Choudghal, Sana Rahman, Mayur Sadar, Ayush Kumar et.al. A Brief Study on Motor Neuron Disease; and its Treatment Includes Drug Delivery System; Future Aspects on Neurological Science" IOSR Journal of Pharmacy and Biological Sciences (IOSR-JPBS) 14.4 (2019): 52-56.DOI:10.9790/3008-1404015256

[2] UmamaYezdani,Mohammad Gayoor khan, ZubiaZainab, Mayur sadar ,Shivam Choudghal, Damini Mishra, Hari Baska. The Current Scenario of Pharmaceutical Research; 3d Tissue Engineering Considered as Eminent Technique. ARC Journal of Public Health and Community Medicine.2019; 4(2):16-22.

[3] Gayoor Khan, Umama Yezdani, Rohit Verma, Raqshan Jabeen, Pradeep Sintha. Detection of Phlebovirus by using qualitative Real time (RT) - PCR and application of silver nanoparticles to control it. World J Pharm Pharm Sci.2018;7(11):936-52.

[4] Mohd.Gayoor Khan.The Novel Drug Delivery System. World J Pharm Pharm Sci. 2017;6(7):477-487.

[5] Unama Yezdani, Mohd. Gayoor Khan, Fazal Khan, Arvind Verma, Nilesh Kushwah, Rohit Verma.The Drug Targeting in Alzheimer's or Applications \& it's Hazards. World J Pharm Sci.2017;7(11):1532-1549.

[6] Dr.H. S Chandel., Sharad P. Panday, Arvind Dangi, Ashish chaurasia, Mohd.Gayoor khan et al. Development of Targeted Drug delivery. International Journal of research methodology Ijrm.Human., 2017 Vol. 1 (2): 30-34.

[7] Kushwah Nilesh, Yezdani Umama, Mohammad Gayoor khan, Manish kushwah, Kumar Ayush.The Fundamental of Novel Drug Delivery System; Methodology, Role of Nanotechnology; Nanoparticles in Pharmaceutical Research. International Journal of Emerging Technologies and Innovative Research (www.jetir.org), ISSN:2349-5162, Vol.6, Issue 6, page no.140-146, June-2019. DOI: http://doi.one/10.1729/Journal.21510

[8] Mohd. Gayoor Khan, Nilesh Kushwaha, Fazal Khan, Vipul Patel. Microencapsulation. International Journal of research methodology Ijrm.Human., 2017 Vol. 1 (2):35-42.

[9] Sai Kiran NA, Vaishya S, Kale SS, Sharma BS, Mahapatra AK. Surgical results in patients with tuberculosis of the spine and severe lower-extremity motor deficits: a retrospective study of 48 patients. J Neurosurg Spine. 2007;6:320-326. [PubMed] [Google Scholar]

[10] Rezai AR, Lee M, Cooper PR, Errico TJ, Koslow M. Modern management of spinal tuberculosis. Neurosurgery. 1995;36:87-97. [PubMed] [Google Scholar]

[11] Turgut M. Spinal tuberculosis (Pott's disease): its clinical presentation, surgical management, and outcome. A survey study on 694 patients. Neurosurg Rev. 2001;24:8-13. [PubMed] [Google Scholar]

[12] Barnes PF, Bloch AB, Davidson PT, Snider DE., Jr Tuberculosis in patients with human immunodeficiency virus infection. N Engl J Med. 1991;324:1644-1650. [PubMed] [Google Scholar]

[13] Moorthy S, Prabhu NK. Spectrum of MR imaging findings in spinal tuberculosis. AJR Am J Roentgenol. 2002;179:979-983. [PubMed] [Google Scholar]

[14] Pertuiset E, Beaudreuil J, Liote F, et al. Spinal tuberculosis in adults, A study of 103 cases in a developed country, 1980-1994. Medicine (Baltimore) 1999;78:309-320. [PubMed] [Google Scholar]

[15] Umama Yezdani Mohammad Gayoor khan, Manish kushwah, Kumar Ayush, Nilesh, Kushwah.The Fundamental of Novel Drug Delivery System; Methodology, Role of Nanotechnology; Nanoparticles in Pharmaceutical Research. International Journal of Emerging Technologies and Innovative Research.6;(6):140-146. https://scholar.google.com/scholar?oi=bibs\&cluster=947193741435004997\&btnI=1\&hl=en\&authuser=1

[16] Umama Yezdani, Mahmood Ali, Mohammad Gayoor Khan,Ayush Kumar, Prince Bhalla and Mayur Sadar, Pharmacological and Non-Pharmacological approaches to Vitiligo, World journal of pharmaceutical research•2019; 8(9):884-892.

Citation: Umama Yezdani, et.al., "Brief Study on Antibiotics Rifampin, Ethambutol, Pyrazinamide. Isoniazid for the Treatment of Tuberculosis Patient; A Combination Therapy, Advanced Techniques. " ARC Journal of Pharmaceutical Sciences (AJPS), 6(1), pp 6-13. DOI: http://dx.doi.org/10.20431/2455-1538.0601002

Copyright: () 2020 Authors. This is an open-access article distributed under the terms of the Creative Commons Attribution License, which permits unrestricted use, distribution, and reproduction in any medium, provided the original author and source are credited. 